\title{
Análisis de la eficacia de los planes de manejo de bosque nativo en la provincia de Chubut, Argentina
}

\author{
Analysis of the effectiveness of native forest management plans in Chubut Province, \\ Patagonia, Argentina
}

FRANCISCO ANDRES CARABELLI, JUAN JOSE FERRANDO

Centro de Investigación y Extensión Forestal Andino-Patagónico (CIEFAP),

C.C. 14 (9200) Esquel, provincia de Chubut, República Argentina.

E-mails: gestion@ciefap.cyt.edu.ar - gomezcarabe@ar.inter.net

\begin{abstract}
SUMMARY
Native forests in Chubut Province cover an area of 1,000,000 hectares, of which 133,000 hectares are considered suitable for timber production. Approximately 10,000 hectares of this productive forest area has been, or is currently, the object of some form of timber exploitation. Pure Nothofagus pumilio forests (known colloquially as "lenga" forests) make up the largest native forest area (90\%) in the designated land. In 1992, specific norms were introduced requiring those who intend to extract native forest timber to present management plans. The management plans presented to the provincial forest authority were analyzed with the aim of assessing their actual effectiveness as administrative tools for promoting the sustainable conservation of forest resources. One of the main conclusions which needs to be highlighted is the remarkable lack of coordination between planning and execution. This arises from: 1) Deficiencies in the structural characterization and volumetric estimation of the forest; 2) Conflicts over resource use, and 3) Conflicts of interests between businessmen and the state.
\end{abstract}

Key words: Patagonia, forest management, native forest.

\section{RESUMEN}

La provincia del Chubut posee, en la zona andina, 1.000.000 de hectáreas de bosques nativos, de las cuales 133.000 hectáreas, mayoritariamente conformadas por bosques puros de Nothofagus pumilio "lenga" (90\%) se consideran aptas para la producción de madera. De ellas, aproximadamente 10.000 hectáreas han sido o son objeto de algún régimen de aprovechamiento forestal. En 1992 se pusieron en vigencia unas normas específicas que básicamente exigen a quienes pretenden extraer madera del bosque nativo la presentación de planes de manejo forestal. Mediante este estudio analizamos planes de manejo presentados a la autoridad forestal de la provincia con el objeto de discutir la eficacia real de estos planes como herramientas para una administración del bosque nativo capaz de promover a mediano y largo plazo la conservación del recurso. Entre los principales resultados debe destacarse que existe una notable falta de correspondencia entre planificación y ejecución, la que se pone en evidencia por: 1) Deficiencias en las caracterizaciones estructurales y en las estimaciones volumétricas; 2) Conflictos en el uso de los recursos, y 3) Conflicto de intereses entre los empresarios y el Estado.

Palabras claves: Patagonia, manejo forestal, bosque nativo. 
BOSQUE 25(1): 3-13, 2004

Análisis de la eficacia de los planes de manejo de bosque nativo en la provincia de Chubut, Argentina

\section{INTRODUCCION}

En 1995 la provincia de Chubut realizó un amplio estudio con el objeto de generar información cuantitativa y cualitativa básica de los bosques considerados "a priori" de producción, para planificar en una etapa posterior la ordenación regional del recurso forestal (1). Del total de superficie boscosa que existe en el territorio provincial en la zona andina, aproximadamente 1.000.000 de hectáreas, 133.000 hectáreas de bosque nativo se clasificaron como productivas para la extracción maderera y de ellas 10.000 hectáreas han sido o son objeto de algún régimen de aprovechamiento forestal.

En el estudio mencionado, el bosque nativo con potencial para la extracción maderera (BNPEM) se definió como aquel del que resulta posible extraer periódicamente productos rollizos o subproductos forestales de valor económico, mediante aprovechamiento racional. La administración técnica del BNPEM se realiza a través del servicio forestal provincial.

Noventa por ciento del BPNEM está conformado por bosques puros de lenga (Nothofagus pumilio Poepp. et Endl. Krasser), mientras que el diez por ciento restante lo constituyen bosques monoespecíficos o mixtos de ciprés de la cordillera (Austrocedrus chilensis D. Don Pic. Serm et Bizarri) y coihue (Nothofagus dombeyi Mirb. Blume). El "rollizo", principal producto que se extrae del BPNEM, se transforma para ser utilizado en madera para carpintería y construcción. Los volúmenes de producción de rollizos fluctúan entre 1.000 y $18.000 \mathrm{~m}^{3} / \mathrm{año}$, siendo el promedio de $9.000 \mathrm{~m}^{3} /$ año. De este volumen bruto se obtienen alrededor de $3.500 \mathrm{~m}^{3}$ /año de madera, de la que veinte por ciento se destina a carpintería, mientras que el ochenta por ciento restante se utiliza en la construcción.

En el año 1992, con el objeto de mejorar las formas de intervenir el BNPEM, se pusieron en vigencia unas Normas para la Elaboración de Planes de Manejo (3), principalmente dirigidas a pautar la forma de elaborar los Planes de Manejo. El horizonte de planificación de estos planes no supera 5 años (4). Los trabajos de aprovechamiento son planificados y ejecutados por los solicitantes privados (generalmente aserraderos). El servicio forestal provincial dispone de un marco adecuado de contención técnica para la toma de decisiones referida a la aprobación de esos planes. Sin embargo, la insuficiente disponibilidad de recursos humanos, infraestructura e insumos para las tareas de control y seguimiento de estos planes de manejo vulneran la efectividad de la fiscalización .

En este contexto, nuestra intención ha sido analizar, a través de los planes de manejo presentados al servicio forestal provincial, la situación actual del uso de bosques en la provincia y discutir la eficacia real de estos planes como herramientas para administrar el bosque nativo y promover a mediano y largo plazo la conservación del recurso y la generación de estructuras más productivas.

\section{MATERIAL Y METODOS}

El área de análisis corresponde a la región andino patagónica de la provincia de Chubut, Argentina, ubicada entre los paralelos $42^{\circ}$ L.S. y $46^{\circ}$ L.S. (figura 1). Inicialmente se consideró la totalidad de planes presentados entre 1992 y 2001. Para cada uno de ellos se examinó la especie, superficie y la instancia administrativa en la que se encontraba, la cual podía ser alguna de las cuatro siguientes: I) en evaluación, II) autorizado, III) en ejecución, IV) ejecutado.

Un conjunto de 12 planes de manejo para bosques de lenga y ciprés de la cordillera fue detalladamente analizado en cada una de sus partes componentes. A ellos se agregaron 13 planes más para ampliar el análisis en relación con el cumplimiento de la norma, con lo que se involucró una porción significativa (25\%) de los planes presentados durante el decenio 1992-2001. Tales planes se seleccionaron de acuerdo con los siguientes criterios: 1) año de presentación, con el objeto de abarcar el período previamente indicado; 2) ubicación geográfica, de modo que estuviesen regularmente distribuidos en el extenso territorio provincial, y 3) estado del trámite administrativo del plan en el servicio forestal, en el sentido que estuviera aprobado.

El análisis de estos planes tuvo por finalidad establecer el grado de correspondencia con las Normas Técnicas precitadas. Para ello se evaluó la forma en que fueron desarrollados los siguientes ítemes:

1) Evaluación del bosque: analizando los datos del inventario y los resultados del mismo. 


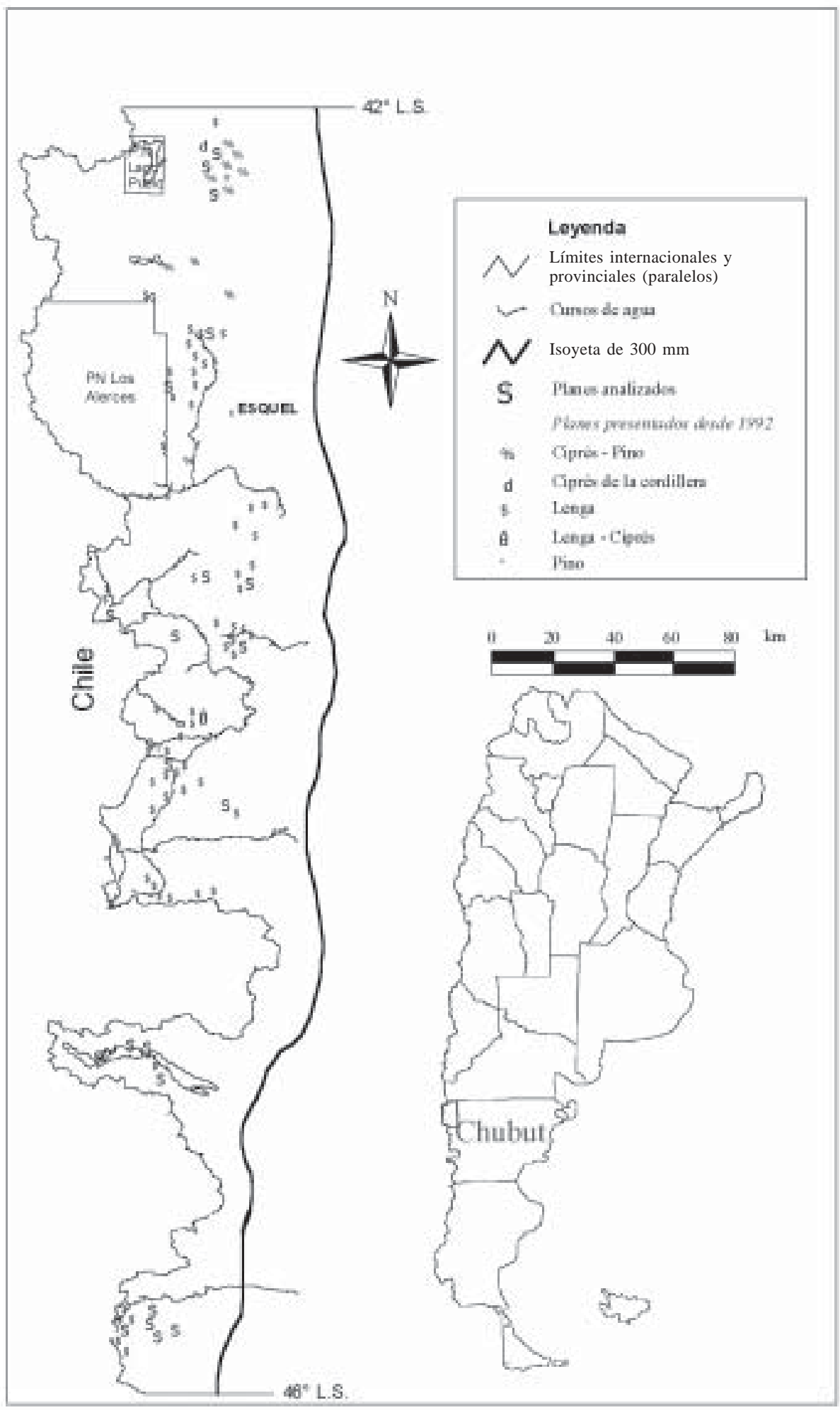

Figura 1. Distribución de los planes de manejo forestal en la región cordillerana de la provincia de Chubut.

Distribution of forest management plans in the Cordillera region of Chubut Province. 
2) Evaluación de la organización económica: examinando los objetivos planteados, el sistema de producción y la organización actual;

3) Planificación del manejo: considerando las metas, la planificación silvícola y la organización del manejo propuestas.

Se estableció una escala nominal de valoración que incluyó cuatro niveles:

a) satisfactorio: cuando el contenido se correspondía plenamente con la exigencia que contemplaba la Norma;

b) regular, cuando la información no era completa, pero sí suficiente para complementar la exigencia que contemplaba la Norma;

c) insuficiente: cuando la información proporcionada era incompleta, y

d) ausente: cuando no se verificó la presencia del ítem.

En relación a la evaluación del bosque se examinaron los parámetros dasométricos relevados, la metodología y el nivel de detalle de los resultados. En el aspecto económico también se estudiaron los costos y rendimientos para cada etapa del aprovechamiento y de la industrialización y cuando ello fue posible se aplicaron indicadores para los análisis de rentabilidad y de sensibilidad. En relación con la planificación silvícola, analizamos las caracterizaciones estructurales, la planificación de los tratamientos silviculturales y la aplicación de los mismos. A base de esta información se analizó la correspondencia entre planificación y ejecución, incluyendo los resultados del control realizado por parte del servicio forestal.

\section{RESULTADOS}

Desde la puesta en vigencia a partir de 1992 de la actual normativa y hasta junio de 2001, se presentaron 99 planes al organismo de control y fiscalización del manejo de bosques (figura 2). Este conjunto de planes cubre una superficie de 17.600 hectáreas, de las cuales 13.300 hectáreas $(75 \%$ de los planes) corresponden a Nothofagus pumilio, 500 hectáreas (3\%) a Austrocedrus chilensis, mientras que 3.800 hectáreas (22\%) involucran una agregación en el mismo plan de bosques de lenga,

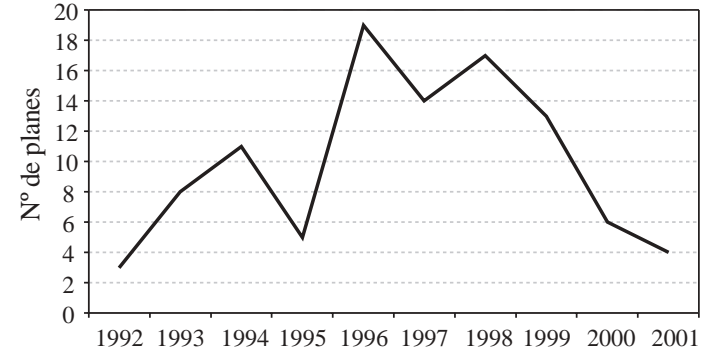

Figura 2. Presentación anual de planes de manejo forestal durante el período 1992-2001.

Annual presentation of forest management plans during the 1990-2001 period.

ciprés y coníferas exóticas. En promedio, la superficie anual intervenida es de 1.100 hectáreas. La variabilidad entre las superficies que involucran los distintos planes es muy alta, pues oscila entre 10 y 1.300 hectáreas.

Desde la instauración de la actual normativa, se manifiesta una tendencia irregular, aunque creciente, de presentación de planes hasta el año 1998. El decrecimiento posterior está vinculado a una disminución del ritmo de la actividad maderera debido a una agobiante situación económica, que se agudizó durante el año 2001.

La superficie anual solicitada en los planes es muy variable, con un mínimo en el año 1995 de 190 hectáreas, probablemente debido al retraso en el inicio de la ejecución de planes anteriores, y un máximo en 1999 de 9.200 hectáreas, que se relaciona con una fuerte presentación de planes de uso múltiple del bosque, en los cuales las superficies con bosque maderero constituyen una porción minoritaria ( $8 \%$ ) de la superficie total involucrada.

Del total de planes presentados, 72 han sido autorizados, mientras que los restantes se encuentran en etapa de evaluación (22) o no han sido autorizados (5). Entre los planes autorizados, 59 (82\%) se hallan ejecutados o en etapa de ejecución y $13(18 \%)$ no han sido ejecutados, a pesar de que en algunos casos han pasado varios años desde su autorización. Para algunos de estos planes se ha podido constatar, aunque no ha sido factible obtener la evidencia directa de ello, que su elaboración por parte de los ocupantes o propietarios de la tierra tiene como principal objetivo impedir que terceros puedan utilizar el vuelo forestal, el que, de acuerdo con la Constitución de la provincia, es propiedad inalienable del Estado. 
El grado de cumplimiento de la norma vigente es muy variable entre planes (figura 3a). Esta comprobación surge del análisis de 21 planes, observándose que $33 \%$ tiene un cumplimiento satisfactorio de los ítemes exigidos en la normativa, mientras que el 67\% restante oscila entre regular (24\%), insuficiente (33\%) y ausente (10\%). Mientras tanto, en la figura $3 \mathrm{~b}$ se ilustra cuál ha sido el nivel de satisfacción en cada uno de los principales rubros que exige la normativa.

En relación con el contenido de la norma, se observa que el mayor apego se verifica en el aspecto referido a la "evaluación dasométrica del bosque", seguido por la información sobre parámetros de rendimiento (cuadro 1). El aspecto más débilmente considerado es el de "evaluación de la organización económica". El análisis económico está en general desprovisto de indicadores financieros. Los casos más elaborados se limitan a hacer una descripción de activos y un detalle de rendimientos y costos por actividades, en forma de una enumeración desprovista de cualquier análisis de la situación actual y de la forma de mejorar la estructura productiva del industrial.

Una descripción sumaria de los resultados de la evaluación dasométrica del bosque sobre un grupo de 12 planes respecto del nivel de satisfacción de los requerimientos de la normativa se presenta en el cuadro 2.

La información que brindan los propios planes (cuadros 3a, 3b y 3c) es en muchos casos incompleta. La relación beneficio/costo, calculada en este

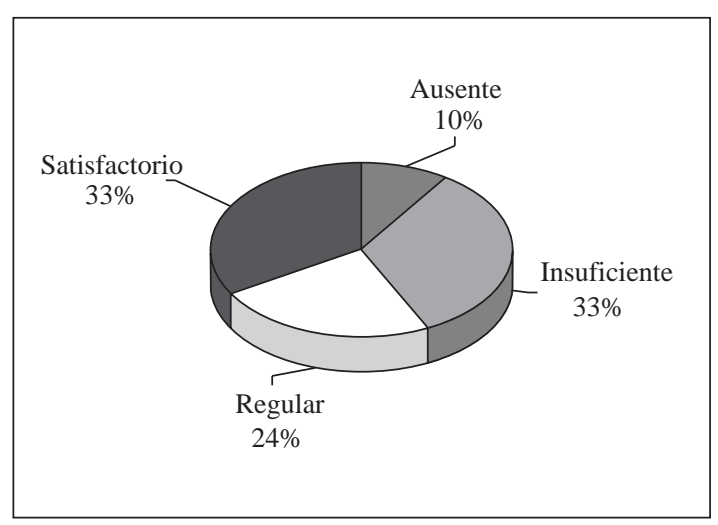

Figura 3a. Valoración general del grado de cumplimiento de la normativa para la elaboración de planes de manejo forestal.

General assessment of the extent to which legal norms are met in the development of forest management plans. estudio para los escasos planes que indican valores de rendimiento, precio ponderado de los productos extraídos y costos -sin tener en cuenta costos posteriores por mantenimiento y tratamientos silvícolas con poca probabilidad de extraer productos comerciales-, es en general muy baja. Esta comprobación permite albergar dudas acerca de la factibilidad económico-financiera que pudieron tener esos emprendimientos. Entre las medidas de "planificación del manejo", la menos atendida es la referida a la organización del mismo, que sólo está presente en un 50\% de los planes analizados. Esto resulta muy significativo cuando se considera que la norma fue concebida como una herramienta para mejorar el estado de conservación y producción de los bosques.

El análisis de la ejecución del plan en relación con el plan en sí mismo (cuadro 4) puso de manifiesto que en términos generales no existe correspondencia entre una instancia y otra. La caracterización analítica resultó una tarea exigente dadas las grandes diferencias que existían entre la información que brindaba la planificación silvícola vertida en los planes y la que se indicaba en los informes de marcación que precedían a la corta. La planificación silvícola sólo informaba, en la mayoría de los casos, acerca del número de árboles, la cantidad de metros cúbicos de rollizos (maderable y total) por rodal que se extraerían y lo que quedaría como remanente -en unos pocos casos acompañado por una estimación estadística del error-. Por su parte, los informes de marcación

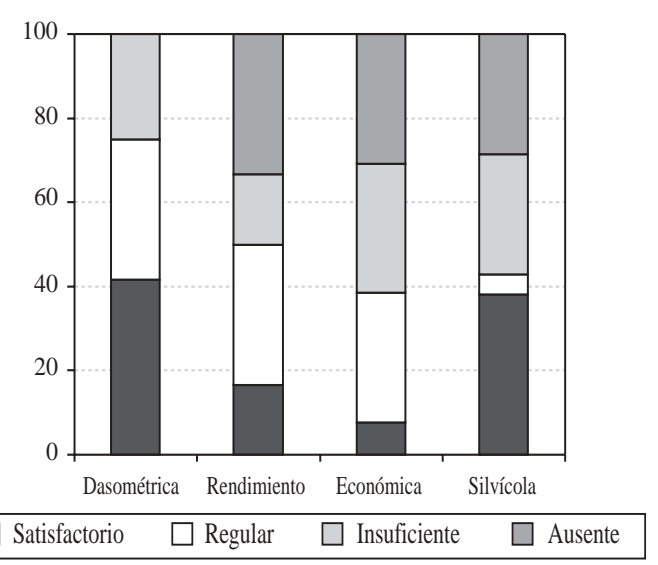

Figura $3 b$. Valoración específica del grado de satisfacción de los distintos rubros técnicos que componen el plan de manejo forestal.

Specific assessment of the success of the different technical sections that make up the forest management plan. 


\section{CUADRO 1}

Niveles de cumplimiento de la normativa para la elaboración de planes de manejo forestal. Levels of accomplishment of the normative to develop forest management plans.

\begin{tabular}{|c|c|c|c|c|c|c|c|c|}
\hline \multirow[b]{2}{*}{$\begin{array}{l}\text { Número } \\
\text { de } \\
\text { Plan }\end{array}$} & \multicolumn{2}{|c|}{$\begin{array}{l}\text { Evaluación } \\
\text { del bosque }\end{array}$} & \multicolumn{3}{|c|}{$\begin{array}{c}\text { Evaluación de la } \\
\text { organización económica }\end{array}$} & \multicolumn{3}{|c|}{$\begin{array}{l}\text { Planificación } \\
\text { del manejo }\end{array}$} \\
\hline & Inventario & Resultados & Objetivos & $\begin{array}{c}\text { Sistema } \\
\text { de } \\
\text { producción }\end{array}$ & $\begin{array}{c}\text { Evaluación de } \\
\text { la organización } \\
\text { actual }\end{array}$ & Metas & $\begin{array}{l}\text { Planificación } \\
\text { Silvícola }\end{array}$ & $\begin{array}{c}\text { Organización } \\
\text { del } \\
\text { manejo }\end{array}$ \\
\hline 1 & $\mathrm{c}$ & $\mathrm{c}$ & $\mathrm{c}$ & $\mathrm{c}$ & $\mathrm{c}$ & $\mathrm{c}$ & $\mathrm{c}$ & $\mathrm{c}$ \\
\hline 2 & $\mathrm{c}$ & $\mathrm{c}$ & $\mathrm{c}$ & s & $\mathrm{i}$ & $\mathrm{c}$ & $\mathrm{c}$ & $\mathrm{c}$ \\
\hline 3 & $\mathrm{c}$ & $\mathrm{c}$ & $\mathrm{c}$ & $\mathrm{c}$ & $\mathrm{c}$ & $\mathrm{c}$ & $\mathrm{c}$ & $\mathrm{c}$ \\
\hline 4 & c & $\mathrm{c}$ & $\mathrm{c}$ & $\mathrm{c}$ & $\mathrm{c}$ & c & $\mathrm{c}$ & $\mathrm{c}$ \\
\hline 5 & $\mathrm{c}$ & $\mathrm{c}$ & c & $\mathrm{a}$ & $\mathrm{a}$ & c & $\mathrm{c}$ & $\mathrm{c}$ \\
\hline 6 & $\mathrm{c}$ & $\mathrm{c}$ & $\mathrm{c}$ & $\mathrm{c}$ & $\mathrm{c}$ & $\mathrm{c}$ & $\mathrm{c}$ & $\mathrm{c}$ \\
\hline 8 & $\mathrm{c}$ & $\mathrm{c}$ & s & $\mathrm{a}$ & $\mathrm{a}$ & $\mathrm{c}$ & $\mathrm{i}$ & $\mathrm{a}$ \\
\hline 11 & $\mathrm{c}$ & s & $\mathrm{a}$ & $\mathrm{a}$ & a & i & s & i \\
\hline 13 & $\mathrm{c}$ & $\mathrm{c}$ & $\mathrm{s}$ & $\mathrm{i}$ & $\mathrm{a}$ & i & c & $\mathrm{a}$ \\
\hline 14 & $\mathrm{c}$ & $\mathrm{c}$ & $\mathrm{c}$ & s & i & $\mathrm{c}$ & $\mathrm{c}$ & $\mathrm{c}$ \\
\hline 15 & $\mathrm{c}$ & $\mathrm{c}$ & $\mathrm{a}$ & $\mathrm{a}$ & a & $\mathrm{c}$ & $\mathrm{i}$ & $\mathrm{a}$ \\
\hline 16 & $\mathrm{c}$ & $\mathrm{c}$ & $\mathrm{a}$ & $\mathrm{a}$ & a & $\mathrm{i}$ & $\mathrm{a}$ & $\mathrm{a}$ \\
\hline 17 & $\mathrm{c}$ & $\mathrm{c}$ & $\mathrm{a}$ & $\mathrm{a}$ & $\mathrm{a}$ & i & $\mathrm{a}$ & $\mathrm{a}$ \\
\hline 18 & $\mathrm{c}$ & $\mathrm{c}$ & $\mathrm{c}$ & $\mathrm{c}$ & $\mathrm{c}$ & $\mathrm{c}$ & $\mathrm{c}$ & $\mathrm{c}$ \\
\hline 19 & $\mathrm{c}$ & $\mathrm{c}$ & $\mathrm{c}$ & $\mathrm{c}$ & $\mathrm{c}$ & $\mathrm{a}$ & $\mathrm{i}$ & $\mathrm{i}$ \\
\hline 20 & $\mathrm{a}$ & $\mathrm{a}$ & $\mathrm{c}$ & $\mathrm{c}$ & $\mathrm{c}$ & $\mathrm{a}$ & $\mathrm{i}$ & $\mathrm{a}$ \\
\hline 21 & $\mathrm{a}$ & $\mathrm{a}$ & $\mathrm{a}$ & $\mathrm{i}$ & $\mathrm{a}$ & $\mathrm{a}$ & s & $\mathrm{a}$ \\
\hline 22 & $\mathrm{c}$ & $\mathrm{c}$ & $\mathrm{c}$ & $\mathrm{i}$ & $\mathrm{a}$ & $\mathrm{a}$ & $\mathrm{c}$ & $\mathrm{a}$ \\
\hline 23 & $\mathrm{c}$ & $\mathrm{s}$ & $\mathrm{s}$ & $\mathrm{a}$ & $\mathrm{a}$ & $\mathrm{a}$ & $\mathrm{i}$ & $\mathrm{a}$ \\
\hline 24 & $\mathrm{c}$ & $\mathrm{c}$ & $c$ & $\mathrm{i}$ & $\mathrm{a}$ & $\mathrm{a}$ & $c$ & $\mathrm{c}$ \\
\hline 25 & $\mathrm{a}$ & $\mathrm{a}$ & $\mathrm{a}$ & $\mathrm{a}$ & $\mathrm{a}$ & $\mathrm{a}$ & s & $\mathrm{a}$ \\
\hline
\end{tabular}

Referencias: $\mathrm{c}=$ completa; $\mathrm{s}=$ suficiente $; \mathrm{i}=$ insuficiente; $\mathrm{a}=$ ausente.

ofrecían datos del número de árboles a extraer, sin discriminar su ubicación, y algunas veces ofrecían datos de volumen. La información cuantitativa acerca del bosque remanente fue casi inexistente. Entre las causas que pueden explicar, al menos parcialmente, esta falta de relación entre planificación y ejecución destacamos las siguientes:

1) Deficiencias en las caracterizaciones estructurales: que se relacionan directamente con debilidades en el cálculo de las existencias maderables y en el diseño de la planificación silvícola.

2) Deficiencias en las estimaciones volumétricas: debidas a muestreos de muy baja intensidad que, por ende, carecen de una mínima representatividad y a las fórmulas de cálculo en sí.

3) Conflictos en el uso de los recursos: en no pocas oportunidades los titulares de los planes contratan a un técnico para que elabore y presente una propuesta de manejo ante el organismo de fiscalización y control con la única intención de evitar que una parte o la totalidad del bosque de su propiedad, o que ocupa con derechos, sea usufructuado por terceros.

4) Conflicto de intereses entre los empresarios y el Estado: en muchos casos es evidente que el empresario no utiliza ni al plan de manejo ni al técnico como herramientas para mejorar la producción, sino sólo como un requisito necesario para cumplir con lo que el Estado le exige, a fin de poder acceder a la explotación del recurso.

De la revisión de los informes de marcación se evidencia que en varios casos la tarea de selección de los árboles que se cortarán está generalmente en manos de los motosierristas o de los mismos madereros, en tanto que la tarea del técnico se 
BOSQUE 25(1): 3-13, 2004

Análisis de la eficacia de los planes de manejo de bosque nativo en la provincia de Chubut, Argentina

\section{CUADRO 2}

Detalle de los principales parámetros para la caracterización dasométrica del bosque.

Details of the main parameters used in the dasometric characterization of the forest.

\begin{tabular}{|c|c|c|c|c|c|c|c|c|c|c|}
\hline $\begin{array}{l}\text { Número } \\
\text { de } \\
\text { Plan }\end{array}$ & $\begin{array}{c}\text { Duración } \\
\text { prevista } \\
\text { [años] }\end{array}$ & Especie & $\begin{array}{c}\text { Superficie } \\
\text { efectiva } \\
\text { [ha] }\end{array}$ & $\begin{array}{c}\text { Vol. mad. } \\
\text { corta } \\
{\left[\mathrm{m}^{3} / \mathrm{ha}\right]}\end{array}$ & $\begin{array}{c}\text { Vol. bruto } \\
\text { corta } \\
{\left[\mathrm{m}^{3} / \mathrm{ha}\right]}\end{array}$ & $\begin{array}{c}\mathrm{N}^{\circ} \text { plantas } \\
\text { corta } \\
\text { [árb/ha] }\end{array}$ & $\begin{array}{c}\text { Vol. mad./ } \\
\text { planta } \\
{\left[\mathrm{m}^{3}\right]}\end{array}$ & $\begin{array}{c}\text { Vol. bruto/ } \\
\text { planta } \\
{\left[\mathrm{m}^{3}\right]}\end{array}$ & $\begin{array}{l}\mathrm{N}^{\circ} \\
\text { trozas } \\
\text { planta }\end{array}$ & $\begin{array}{c}\text { Vol. medio/ } \\
\text { troza } \\
{\left[\mathrm{m}^{3}\right]}\end{array}$ \\
\hline 1 & 1 & Lenga & 24 & 27 & 200 & 170 & 0,5 & 1,2 & 1,2 & 0,4 \\
\hline 2 & 5 & Lenga & 290 & 18 & 328 & 300 & 0,94 & 1,09 & $\mathrm{~s} / \mathrm{d}$ & $\mathrm{s} / \mathrm{d}$ \\
\hline 3 & 5 & Lenga & 121 & 60 & 372 & 284 & 1,01 & 1,3 & 1,5 & 0,67 \\
\hline 4 & 5 & Lenga & 59 & 60 & 281 & 174 & 1,18 & 1,61 & 1,5 & 0,78 \\
\hline 5 & 1 & Lenga & 18,5 & 70 & 407 & 100 & 2,55 & 4,07 & s/d & $\mathrm{s} / \mathrm{d}$ \\
\hline 6 & 2 & Lenga & 84 & 59 & 193 & 136 & 1,09 & 1,42 & 1,5 & 0,72 \\
\hline 7 & 1 & Lenga & 38 & 25 & 120 & 158 & $\mathrm{~s} / \mathrm{d}$ & 0,764 & 1,5 & 0,3 \\
\hline 8 & 1 & Lenga & 46 & 44,5 & 449 & 130 & $\mathrm{~s} / \mathrm{d}$ & 3,46 & 1,4 & $\mathrm{~s} / \mathrm{d}$ \\
\hline 9 & 5 & $\begin{array}{c}\text { Ciprés y } \\
\text { otras }\end{array}$ & 63 & 161 & 232 & 128 & $\mathrm{~s} / \mathrm{d}$ & $\mathrm{s} / \mathrm{d}$ & $\mathrm{s} / \mathrm{d}$ & $\mathrm{s} / \mathrm{d}$ \\
\hline 10 & 10 & Ciprés & 30 & 70 & 120 & 68 & 2,06 & 2,86 & $\mathrm{~s} / \mathrm{d}$ & $\mathrm{s} / \mathrm{d}$ \\
\hline 11 & 5 & $\begin{array}{l}\text { Ciprés- } \\
\text { Coihue }\end{array}$ & 54,5 & 59 & $\mathrm{~s} / \mathrm{d}$ & 125 & 0,47 & $\mathrm{~s} / \mathrm{d}$ & $\mathrm{s} / \mathrm{d}$ & s/d \\
\hline 12 & 10 & Ciprés & 18 & $\mathrm{~s} / \mathrm{d}$ & 95 & 213 & $\mathrm{~s} / \mathrm{d}$ & 0,446 & $\mathrm{~s} / \mathrm{d}$ & $\mathrm{s} / \mathrm{d}$ \\
\hline
\end{tabular}

\section{CUADRO 3a}

Detalle de la información sobre rendimiento incluida en los planes de manejo forestal. Details of yield information included in forest resource management plans.

\begin{tabular}{|c|c|c|c|c|c|c|c|c|c|c|c|c|}
\hline \multirow{3}{*}{$\begin{array}{l}\mathrm{N}^{\circ} \\
\text { Plan }\end{array}$} & \multicolumn{9}{|c|}{ Rendimiento en el aprovechamiento } & \multicolumn{3}{|c|}{ Rend. industrial } \\
\hline & \multicolumn{2}{|c|}{ Apeo } & \multicolumn{2}{|c|}{ Trozado } & \multicolumn{3}{|c|}{ Arrastre } & \multirow{2}{*}{$\begin{array}{c}\text { Carga } \\
{\left[\mathrm{m}^{3} /\right.} \\
\text { camión }]\end{array}$} & \multirow{2}{*}{$\begin{array}{c}\text { Transporte } \\
\text { [m³/día] }\end{array}$} & \multirow{2}{*}{$\begin{array}{c}\text { Capacidad } \\
\text { Potencial } \\
\text { [pº́día] }\end{array}$} & \multirow{2}{*}{$\begin{array}{c}\text { Capacidad } \\
\text { Actual } \\
\text { [p²/día] }\end{array}$} & \multirow{2}{*}{$\begin{array}{c}\text { Rendimiento } \\
{\left[\mathrm{p}^{2} / \mathrm{m}^{3}\right]}\end{array}$} \\
\hline & [p/día] & [m³/día] & [pl/día] & [m³/día] & $\begin{array}{c}\text { Dist. } \\
\text { media }[\mathrm{m}]\end{array}$ & $\begin{array}{c}{\left[\mathrm{N}^{\circ} \text { trozas } /\right.} \\
\text { Jornal }]\end{array}$ & $\begin{array}{c}\mathrm{m}^{3} / \mathrm{dí}^{\prime} / \\
\text { yunta] }\end{array}$ & & & & & \\
\hline 1 & 50 & 48 & 20 & 19 & 800 (B) & 25 & 10 & 10 & 120 & $\begin{array}{l}5.000- \\
6.000\end{array}$ & $\begin{array}{l}2.700- \\
2.800\end{array}$ & 140 \\
\hline 2 & 100 & S/d & 50 & $\mathrm{~s} / \mathrm{d}$ & 150 (B) & 40 & $\mathrm{~s} / \mathrm{d}$ & $\begin{array}{c}6,5 \text { a } 12 \\
M / 18 \mathrm{~L}\end{array}$ & $\mathrm{~s} / \mathrm{d}$ & 4.000 & 2.000 & $\mathrm{~s} / \mathrm{d}$ \\
\hline 3 & $\mathrm{~s} / \mathrm{d}$ & $90(+\mathrm{T})$ & $\mathrm{s} / \mathrm{d}$ & $\mathrm{s} / \mathrm{d}$ & $300(\operatorname{Tr})$ & $\mathrm{s} / \mathrm{d}$ & 50 & $190 \mathrm{~m}^{3} /$ día & 140 & 1.200 & Sin instalar & 180 \\
\hline 4 & $60(+\mathrm{T})$ & $\mathrm{s} / \mathrm{d}$ & $\mathrm{s} / \mathrm{d}$ & $\mathrm{s} / \mathrm{d}$ & 200 (B) & 30 & $\mathrm{~s} / \mathrm{d}$ & 10 & 20 & 3.200 & 630 & 140 \\
\hline 5 & $\begin{array}{c}100(35- \\
50+\mathrm{T})\end{array}$ & s/d & s/d & $\mathrm{s} / \mathrm{d}$ & 100 (B) & $s / d$ & $\mathrm{~s} / \mathrm{d}$ & $6-7$ & 14 & 1.500 & Sin instalar & 140 \\
\hline 6 & $30(+\mathrm{T})$ & 29 & $\begin{array}{c}30(40- \\
50 \mathrm{r})\end{array}$ & 23 & 200 (B) & $25-30$ & $12-15$ & 6 & 48 & 1.540 & 1.284 & 130 \\
\hline 7 & $70(+\mathrm{T})$ & $\mathrm{s} / \mathrm{d}$ & $\mathrm{s} / \mathrm{d}$ & $\mathrm{s} / \mathrm{d}$ & 50 (B) & $\mathrm{s} / \mathrm{d}$ & 25 & 14 & $\mathrm{~s} / \mathrm{d}$ & $\mathrm{s} / \mathrm{d}$ & $\mathrm{s} / \mathrm{d}$ & 150 \\
\hline 8 & $50(+\mathrm{T})$ & s/d & $70 \mathrm{r}$ & $s / d$ & 150 (B) & $s / d$ & 30 & $7-8$ & 37,5 & $s / d$ & $s / d$ & 120 \\
\hline 9 & $\mathrm{~s} / \mathrm{d}$ & s/d & s/d & $\mathrm{s} / \mathrm{d}$ & 200 (B) & $s / d$ & $\mathrm{~s} / \mathrm{d}$ & $\mathrm{s} / \mathrm{d}$ & $\mathrm{s} / \mathrm{d}$ & $s / d$ & $\mathrm{~s} / \mathrm{d}$ & $\mathrm{s} / \mathrm{d}$ \\
\hline 10 & $\mathrm{~s} / \mathrm{d}$ & s/d & $\mathrm{s} / \mathrm{d}$ & $\mathrm{s} / \mathrm{d}$ & $30-40$ (B) & $\mathrm{s} / \mathrm{d}$ & $\mathrm{s} / \mathrm{d}$ & $\mathrm{s} / \mathrm{d}$ & $\mathrm{s} / \mathrm{d}$ & $\mathrm{s} / \mathrm{d}$ & $\mathrm{s} / \mathrm{d}$ & 160 \\
\hline 11 & $\mathrm{~s} / \mathrm{d}$ & $\mathrm{s} / \mathrm{d}$ & $\mathrm{s} / \mathrm{d}$ & $\mathrm{s} / \mathrm{d}$ & $\mathrm{s} / \mathrm{d}$ & $\mathrm{s} / \mathrm{d}$ & s/d & $\mathrm{s} / \mathrm{d}$ & s/d & $\mathrm{s} / \mathrm{d}$ & s/d & 180 \\
\hline 12 & $10(\mathrm{D}+\mathrm{T})$ & $\mathrm{s} / \mathrm{d}$ & $\mathrm{s} / \mathrm{d}$ & $\mathrm{s} / \mathrm{d}$ & 60 (B) & 60 & $\mathrm{~s} / \mathrm{d}$ & $\mathrm{s} / \mathrm{d}$ & $\mathrm{s} / \mathrm{d}$ & $\mathrm{s} / \mathrm{d}$ & $\mathrm{s} / \mathrm{d}$ & $\mathrm{s} / \mathrm{d}$ \\
\hline
\end{tabular}

Referencias: s/d: sin datos; T: trozado; D: descortezado; pl: plantas; r: rollizo; B: bueyes; M: maderable; L: leña. 
BOSQUE 25(1): 3-13, 2004

Análisis de la eficacia de los planes de manejo de bosque nativo en la provincia de Chubut, Argentina

\section{CUADRO $3 b$}

Detalle de la información sobre costos involucrados en las tareas de aprovechamiento.

Financial data for forest exploitation tasks.

\begin{tabular}{|c|c|c|c|c|c|c|c|c|c|c|c|}
\hline \multirow{3}{*}{$\begin{array}{c}\mathrm{N}^{\circ} \\
\text { Plan }\end{array}$} & \multicolumn{11}{|c|}{ Costos de aprovechamiento } \\
\hline & \multicolumn{2}{|c|}{ Apeo } & \multicolumn{2}{|c|}{ Trozado } & \multicolumn{2}{|c|}{ Arrastre } & \multirow{2}{*}{$\begin{array}{c}\text { Carga } \\
{\left[\mathrm{USS} / \mathrm{m}^{3}\right]}\end{array}$} & \multicolumn{2}{|c|}{ Transporte $\left[\mathrm{U} \$ \mathrm{~S} / \mathrm{m}^{3}\right]$} & \multirow{2}{*}{\begin{tabular}{c|} 
Mantenimiento \\
Caminos \\
{$\left[\mathrm{USS} / \mathrm{m}^{3}\right]$}
\end{tabular}} & \multirow{2}{*}{$\begin{array}{c}\text { Aforo } \\
{\left[\mathrm{U} \$ \mathrm{~S} / \mathrm{m}^{3}\right]}\end{array}$} \\
\hline & {$[\mathrm{U} \$ \mathrm{~S} / \mathrm{pl}]$} & {$\left[\mathrm{U} \$ \mathrm{~S} / \mathrm{m}^{3}\right]$} & [U\$S/troza] & {$\left[\mathrm{U} \$ \mathrm{~S} / \mathrm{m}^{3}\right]$} & $\begin{array}{l}\text { USS/ } \\
\text { troza] }\end{array}$ & {$\left[\mathrm{U} \$ \mathrm{~S} / \mathrm{m}^{3}\right]$} & & $\begin{array}{c}\text { Playa } \\
\text { Intermedia }\end{array}$ & $\begin{array}{l}\text { Playa } \\
\text { Final }\end{array}$ & & \\
\hline 1 & 1 & 2,11 & 1,5 & 1,09 & 1,5 & 1,09 & $s / d$ & 0,32 & $\mathrm{~s} / \mathrm{d}$ & 30,59 & 4,41 \\
\hline 2 & $\mathrm{~s} / \mathrm{d}$ & 1,43 & s/d & 1,43 & s/d & 5,22 & 1,21 & $\mathrm{~s} / \mathrm{d}$ & $\mathrm{s} / \mathrm{d}$ & s/d & $\mathrm{s} / \mathrm{d}$ \\
\hline 3 & $\mathrm{~s} / \mathrm{d}$ & $0,85(+\mathrm{T})$ & $s / d$ & S/d & $s / d$ & 2,87 & 1,5 & 3,5 & 5 & $\mathrm{~s} / \mathrm{d}$ & 4,41 \\
\hline 4 & 0,6 & $0,9(+\mathrm{T})$ & 0,6 & s/d & $\mathrm{s} / \mathrm{d}$ & 3,28 & 6 & $\mathrm{~s} / \mathrm{d}$ & 15 & $\mathrm{~s} / \mathrm{d}$ & 4,41 \\
\hline 5 & $S / d$ & $2,86(+\mathrm{T})$ & $\mathrm{s} / \mathrm{d}$ & $\mathrm{s} / \mathrm{d}$ & $\mathrm{s} / \mathrm{d}$ & 5,03 & $\mathrm{~s} / \mathrm{d}$ & $\mathrm{s} / \mathrm{d}$ & $\begin{array}{l}2 \text { (Tab.)/ } \\
0,66(\mathrm{~L})\end{array}$ & $\mathrm{s} / \mathrm{d}$ & 4,41 \\
\hline 6 & $3(+\mathrm{T})$ & $1,6(+\mathrm{T})$ & s/d & $\mathrm{s} / \mathrm{d}$ & 0,7 & 1,85 & 7,7 & s/d & s/d & s/d & 4,41 \\
\hline 7 & $2(+\mathrm{T})$ & $0,9(+\mathrm{T})$ & $\mathrm{s} / \mathrm{d}$ & $s / d$ & 1,3 & 0,39 & $\mathrm{~s} / \mathrm{d}$ & $\mathrm{s} / \mathrm{d}$ & $\begin{array}{c}3,675 \\
\text { (Elabor.) }\end{array}$ & $\mathrm{s} / \mathrm{d}$ & $\mathrm{s} / \mathrm{d}$ \\
\hline 8 & $0,6(\mathrm{~A})$ & & & 5 (incluye & & & & $\mathrm{s} / \mathrm{d}$ & $\mathrm{s} / \mathrm{d}$ & $\mathrm{s} / \mathrm{d}$ & $\mathrm{s} / \mathrm{d}$ \\
\hline 9 & $\mathrm{~s} / \mathrm{d}$ & 8,13 & $(+\mathrm{T}) \mathrm{M} / 2,38$ & & $\mathrm{~s} / \mathrm{d}$ & $4 \mathrm{M} / 2,33 \mathrm{~L}$ & $s / d$ & $\mathrm{~s} / \mathrm{d}$ & $\mathrm{s} / \mathrm{d}$ & $\mathrm{s} / \mathrm{d}$ & $\mathrm{s} / \mathrm{d}$ \\
\hline 10 & $\mathrm{~s} / \mathrm{d}$ & 9,15 & $(+\mathrm{T}) \mathrm{M} / 5,77$ & & $\mathrm{~s} / \mathrm{d}$ & $0,87 \mathrm{M} / 1,46 \mathrm{~L}$ & $\mathrm{~s} / \mathrm{d}$ & $\mathrm{s} / \mathrm{d}$ & 1,13 & $2,78(\mathrm{Co})$ & $\mathrm{s} / \mathrm{d}$ \\
\hline 11 & $\mathrm{~s} / \mathrm{d}$ & $4,5(+\mathrm{T})$ & s/d & s/d & $\mathrm{s} / \mathrm{d}$ & 6 & $\begin{array}{r}2 \text { (acondici } \\
\text { y q }\end{array}$ & $\begin{array}{l}\text { nado ramas } \\
\text { ema) }\end{array}$ & 10 & s/d & $\mathrm{s} / \mathrm{d}$ \\
\hline 12 & $\mathrm{~s} / \mathrm{d}$ & $6(+T)$ & $\mathrm{s} / \mathrm{d}$ & $\mathrm{s} / \mathrm{d}$ & $\mathrm{s} / \mathrm{d}$ & $\mathrm{s} / \mathrm{d}$ & $\mathrm{s} / \mathrm{d}$ & $\mathrm{s} / \mathrm{d}$ & $\mathrm{s} / \mathrm{d}$ & $\mathrm{s} / \mathrm{d}$ & $\mathrm{s} / \mathrm{d}$ \\
\hline
\end{tabular}

Referencias: pl: planta; s/d: sin datos; T: trozado; A: apeo; M: maderable; L: leña; Tab.: tablas.

\section{CUADRO 3c}

Detalle de la información sobre costos involucrados en las tareas de industrialización y comercialización.

Financial data for industrialization and commercialization tasks.

\begin{tabular}{|c|c|c|c|c|c|c|c|c|c|}
\hline \multirow[b]{2}{*}{$\begin{array}{c}\mathrm{N}^{\circ} \\
\text { Plan }\end{array}$} & \multicolumn{4}{|c|}{ Costo de industrializacion } & \multicolumn{2}{|c|}{ Costo comercialización } & \multirow{2}{*}{$\begin{array}{c}\text { Costo } \\
\text { Total } \\
{[\mathrm{U} \$ \mathrm{~S}] / \mathrm{m}^{3}}\end{array}$} & \multirow{2}{*}{$\begin{array}{c}\text { Precio } \\
\text { Ponderado } \\
{\left[\mathrm{U} \$ \mathrm{~S} / \mathrm{p}^{2}\right]}\end{array}$} & \multirow{2}{*}{$\begin{array}{c}\text { Relación } \\
\text { Beneficio /Costo }\end{array}$} \\
\hline & $\begin{array}{l}\text { Personal } \\
{[\mathrm{U} \$ S] / \mathrm{m}^{3}}\end{array}$ & $\begin{array}{c}\text { Amortización } \\
{[\mathrm{U} \$ \mathrm{~S}] / \mathrm{m}^{3}}\end{array}$ & $\begin{array}{c}\text { Elaboración } \\
{[\mathrm{U} \$ S] / \mathrm{m}^{3}}\end{array}$ & $\begin{array}{l}\text { C. Variables } \\
{[\mathrm{U} \$ \mathrm{~S}] / \mathrm{m}^{3}}\end{array}$ & $\begin{array}{c}\text { Personal } \\
{[\mathrm{U} \$ \mathrm{~S}] / \mathrm{m}^{3}}\end{array}$ & $\begin{array}{c}\text { Sin especificar } \\
{[\mathrm{U} \$ \mathrm{~S}] / \mathrm{m}^{3}}\end{array}$ & & & \\
\hline 1 & 5,44 & 3,43 & $\mathrm{~s} / \mathrm{d}$ & 1,82 & 5,28 & $\mathrm{~s} / \mathrm{d}$ & 58,59 & 0,9 & 2,15 \\
\hline 2 & $\mathrm{~s} / \mathrm{d}$ & $\mathrm{s} / \mathrm{d}$ & $s / d$ & $\mathrm{~s} / \mathrm{d}$ & $\mathrm{s} / \mathrm{d}$ & $\mathrm{s} / \mathrm{d}$ & $\mathrm{s} / \mathrm{d}$ & $s / d$ & $\mathrm{~s} / \mathrm{d}$ \\
\hline 3 & 31,43 & 1,35 & 3,59 & 4,79 & $\mathrm{~s} / \mathrm{d}$ & 8,26 & 67,8 & 0,858 & 2,28 \\
\hline 4 & 6,22 & 0,045 & $\mathrm{~s} / \mathrm{d}$ & 1,8 & 2 & 1,32 & 54,52 & 0,534 & 1,37 \\
\hline 5 & $\mathrm{~s} / \mathrm{d}$ & $\mathrm{s} / \mathrm{d}$ & $\mathrm{s} / \mathrm{d}$ & 1,09 & $\mathrm{~s} / \mathrm{d}$ & $\mathrm{s} / \mathrm{d}$ & 14,05 & 0,75 & 7,47 \\
\hline 6 & 109 & $\mathrm{~s} / \mathrm{d}$ & $\mathrm{s} / \mathrm{d}$ & 8,45 & 9,78 & $\mathrm{~s} / \mathrm{d}$ & 177 & 0,55 & 0,40 \\
\hline 7 & 23 & $\mathrm{~s} / \mathrm{d}$ & $\mathrm{s} / \mathrm{d}$ & 1,598 & $\mathrm{~s} / \mathrm{d}$ & $\mathrm{s} / \mathrm{d}$ & 29,41 & $\mathrm{~s} / \mathrm{d}$ & $\mathrm{s} / \mathrm{d}$ \\
\hline 8 & 15,88 & 0,31 & 3,98 & 22,7 & 3,49 & 3,66 & 76,72 & $\mathrm{~s} / \mathrm{d}$ & $\mathrm{s} / \mathrm{d}$ \\
\hline 9 & $\mathrm{~s} / \mathrm{d}$ & $\mathrm{s} / \mathrm{d}$ & $s / d$ & $\mathrm{~s} / \mathrm{d}$ & $\mathrm{s} / \mathrm{d}$ & $\mathrm{s} / \mathrm{d}$ & $\mathrm{s} / \mathrm{d}$ & $s / d$ & $\mathrm{~s} / \mathrm{d}$ \\
\hline 10 & $s / d$ & $\mathrm{~s} / \mathrm{d}$ & $\mathrm{s} / \mathrm{d}$ & $\mathrm{s} / \mathrm{d}$ & $\mathrm{s} / \mathrm{d}$ & $s / d$ & $\mathrm{~s} / \mathrm{d}$ & $\mathrm{s} / \mathrm{d}$ & $\mathrm{s} / \mathrm{d}$ \\
\hline 11 & \multicolumn{4}{|c|}{36 (incluye todos los rubros) } & $\mathrm{s} / \mathrm{d}$ & $\mathrm{s} / \mathrm{d}$ & 88,2 & 0,7 & 1,43 \\
\hline 12 & $\mathrm{~s} / \mathrm{d}$ & $\mathrm{s} / \mathrm{d}$ & $\mathrm{s} / \mathrm{d}$ & $\mathrm{s} / \mathrm{d}$ & $\mathrm{s} / \mathrm{d}$ & $\mathrm{s} / \mathrm{d}$ & $\mathrm{s} / \mathrm{d}$ & $\begin{array}{c}30 / \mathrm{m}^{3} \mathrm{M} / 10 \mathrm{~m}^{3} \mathrm{~L} \\
7,5 \mathrm{pt} / 2,5 \mathrm{pa}\end{array}$ & $\mathrm{s} / \mathrm{d}$ \\
\hline
\end{tabular}

Referencias: pl: planta; s/d: sin datos; T: trozado; A: apeo; M: maderable; L: leña. 


\section{CUADRO 4}

Correspondencia entre memoria técnica y ejecución del plan. Comparison between technical reports and plan execution.

\begin{tabular}{|c|c|c|c|c|c|c|c|c|c|c|}
\hline \multirow{2}{*}{$\begin{array}{l}\text { Número } \\
\text { de } \\
\text { Plan }\end{array}$} & \multirow{2}{*}{$\begin{array}{c}\text { Duración } \\
\text { Prevista } \\
\text { [años] }\end{array}$} & \multirow[b]{2}{*}{ Especie } & \multicolumn{4}{|c|}{ Planificación silvícola } & \multicolumn{4}{|c|}{ Ejecución } \\
\hline & & & $\begin{array}{c}\text { Superficie } \\
\text { Efectiva } \\
\text { [ha] }\end{array}$ & $\begin{array}{c}\text { Vol. Mad. } \\
\text { Corta } \\
{\left[\mathrm{m}^{3} / \mathrm{ha}\right]}\end{array}$ & $\begin{array}{c}\text { Vol. Bruto } \\
\text { Corta } \\
{\left[\mathrm{m}^{3} / \mathrm{ha}\right]}\end{array}$ & $\begin{array}{c}\mathrm{N}^{\circ} \text { Plantas } \\
\text { Corta } \\
\text { [árb/ha] }\end{array}$ & $\begin{array}{c}\text { Superficie } \\
\text { Efectiva } \\
\text { [ha] }\end{array}$ & $\begin{array}{c}\text { Vol. Mad. } \\
\text { Corta } \\
{\left[\mathrm{m}^{3} / \mathrm{ha}\right]}\end{array}$ & $\begin{array}{c}\text { Vol. Bruto } \\
\text { Corta } \\
{\left[\mathrm{m}^{3} / \mathrm{ha}\right]}\end{array}$ & $\begin{array}{c}\mathrm{N}^{\circ} \text { Plantas } \\
\text { Corta } \\
\text { [árb/ha] }\end{array}$ \\
\hline 4 & 5 & Lenga & 59 & 60 & 281 & 174 & 6 & 36 & 111 & 116 \\
\hline 8 & 1 & Lenga & 46 & 44,5 & 449 & 130 & $\mathrm{~s} / \mathrm{d}$ & $\mathrm{s} / \mathrm{d}$ & $\mathrm{s} / \mathrm{d}$ & 132 \\
\hline 11 & 5 & Ciprés-Coihue & 54,5 & 59 & $\mathrm{~s} / \mathrm{d}$ & 125 & 46,5 & 52,84 & $\mathrm{~s} / \mathrm{d}$ & $\mathrm{s} / \mathrm{d}$ \\
\hline 13 & 5 & Lenga & 52 & 13,76 & 715,54 & 80 & 24 & 8,53 & $\mathrm{~s} / \mathrm{d}$ & 9,5 \\
\hline 15 & 1 & Lenga & 74,98 & 32,5 & $\mathrm{~s} / \mathrm{d}$ & 107 & $\mathrm{~s} / \mathrm{d}$ & 20,56 & $\mathrm{~s} / \mathrm{d}$ & 59 \\
\hline 16 & 1 & Lenga & 44 & s/d & $\mathrm{s} / \mathrm{d}$ & $\mathrm{s} / \mathrm{d}$ & $\mathrm{s} / \mathrm{d}$ & $\mathrm{s} / \mathrm{d}$ & $\mathrm{s} / \mathrm{d}$ & 30 \\
\hline 19 & 1 & Lenga & 26,5 & 30,6 & 120,18 & 64 & 26,5 & 44,31 & $\mathrm{~s} / \mathrm{d}$ & 123 \\
\hline 20 & 1 & Lenga & 30,01 & 42,71 & 99,82 & 54 & $\mathrm{~s} / \mathrm{d}$ & $\mathrm{s} / \mathrm{d}$ & $\mathrm{s} / \mathrm{d}$ & 54 \\
\hline 21 & $\mathrm{~s} / \mathrm{d}$ & Lenga & 198 & 41 & 91 & 60 & 198 & 18 & $\mathrm{~s} / \mathrm{d}$ & 22 \\
\hline 22 & 1 & Lenga & 69,3 & $\mathrm{~s} / \mathrm{d}$ & $\mathrm{s} / \mathrm{d}$ & $\mathrm{s} / \mathrm{d}$ & $\mathrm{s} / \mathrm{d}$ & $\mathrm{s} / \mathrm{d}$ & $\mathrm{s} / \mathrm{d}$ & 31 \\
\hline 23 & $\mathrm{~s} / \mathrm{d}$ & Lenga & 121 & 41,48 & $\mathrm{~s} / \mathrm{d}$ & 43 & 121 & 32,2 & $\mathrm{~s} / \mathrm{d}$ & $\mathrm{s} / \mathrm{d}$ \\
\hline 24 & $\mathrm{~s} / \mathrm{d}$ & Lenga & 84,7 & 35,46 & 301,58 & 100 & 84,7 & $\mathrm{~s} / \mathrm{d}$ & $\mathrm{s} / \mathrm{d}$ & 33 \\
\hline 25 & $\mathrm{~s} / \mathrm{d}$ & Lenga & 23 & $\mathrm{~s} / \mathrm{d}$ & $\mathrm{s} / \mathrm{d}$ & 32 & $\mathrm{~s} / \mathrm{d}$ & $\mathrm{s} / \mathrm{d}$ & $\mathrm{s} / \mathrm{d}$ & 57 \\
\hline
\end{tabular}

Referencias: s/d: sin datos. Nota: el número de plan se corresponde con el listado en la cuadro 1.

limita a revisar a posteriori el "tratamiento" aplicado y proponer la corta o anillado del resto de los árboles para que la corta definitiva se asimile tanto como sea posible a la propuesta silvícola contenida en el plan original.

\section{DISCUSION}

Desde principios del siglo XX hasta la actualidad la referencia a la baja productividad de nuestros bosques nativos ha sido una constante. En tal sentido, varios autores $(5,6,7)$ han coincidido en afirmar que el problema forestal más vital para los bosques patagónicos radicaba en su transformación en forestas de mayor valor económico.

A esta apreciación se suma otro problema que es causa permanente de conflictos: los ocupantes ganaderos no quieren explotaciones forestales en sus predios. Teniendo en cuenta que la actividad ganadera ha sido históricamente preponderante, el ocupante, adjudicatario o propietario de la tierra, normalmente heredero de esta tradición, percibe negativamente la presencia del obrajero forestal, pues considera al bosque como un lugar con pastos y que ofrece cierto refugio a los animales. Con esta perspectiva, la presencia de extraños que usufructúan una zona que es de su propiedad, o que ocupa con derechos, conspira contra la cuota de responsabilidad que le corresponde en el cuidado de un recurso tan valioso como frágil. Esta situación de inseguridad en la posesión unificada del suelo y del bosque provee pocos incentivos para medidas de conservación y manejo, y resulta en una degradación del recurso (8). En esta dirección, el Estado debe asumir la responsabilidad que le corresponde en la generación y/o promoción de formas más integradoras de utilización de los recursos forestales que respeten la idiosincrasia de los distintos usuarios.

Finalmente, un tercer aspecto de relevancia es el que se refiere a la industria del aserrío, que, salvo escasas excepciones, no ha desarrollado una estrategia empresarial basada en la búsqueda y captura de mercados para sus productos, los que, por otra parte, poseen muy poco valor agregado como resultado de un proceso de industrialización que utiliza tecnología muy precaria. En el caso de la lenga, que como se ha señalado es la especie con mayor superficie sujeta a planes de manejo (75\% de la superficie total), Tiling y Jovanovski (9) señalan que la industria productora de madera 
BOSQUE 25(1): 3-13, 2004

Análisis de la eficacia de los planes de manejo de bosque nativo en la provincia de Chubut, Argentina

de esta especie ocupa en Chubut una posición débil

frente a las fuerzas competidoras, tales como el ingreso de nuevas firmas al sector, el poder de negociación de los compradores, el poder de negociación de los proveedores, el riesgo de los productos sustitutos y la intensidad de la competencia entre industrias. Agregan que la primitiva tecnología y la falta de capital son los mayores obstáculos para implementar planes de comercialización que tengan en cuenta aspectos tales como el ciclo producto-vida, el posicionamiento, el producto, el precio, el lugar (distribución), la promoción y la gente.

Ante este panorama cabe preguntarse si los planes de manejo, como están actualmente concebidos en sus facetas de elaboración y aplicación, son de utilidad para mejorar la calidad del bosque actual y futuro. La respuesta es incierta. Por un lado, estos planes tienen, en general y como se ha mostrado, numerosas deficiencias de orden técnico que invalidan o limitan, en muchos casos severamente, su aplicación y el consecuente manejo del bosque conforme a pautas silvícolas. En efecto, la evaluación del bosque presenta debilidades en la estimación de los volúmenes maderables en pie (sobre todo para lenga), pero aún considerando que en algunos casos esta estimación pueda tener un grado aceptable de confiabilidad, no considera, generalmente, las modalidades de trozado vigentes y los productos (principalmente piezas largas) que genera cada aserradero, con lo cual se produce un desfasaje que afecta la rentabilidad. Sumado a ello, nuestro análisis también pone en evidencia que las consideraciones económicas son en la mayoría de los planes de manejo sumamente precarias, pues sólo se limitan, en los casos más elaborados, a hacer una descripción de activos y a detallar rendimientos y costos por actividades, en forma de una enumeración desprovista de cualquier análisis de la situación actual y de la forma de mejorar la estructura productiva del industrial. Complementariamente, los planes carecen de un elemental análisis financiero -sobre todo cuando se trata de planes de corta para varios años- que mediante indicadores de rentabilidad le brinden al empresario una base medianamente sólida para evaluar la conveniencia de, por ejemplo, realizar inversiones en compra de maquinarias, tanto para el aserradero como para las tareas de aprovechamiento, de capacitar adecuadamente a sus empleados y, sobre todas las cosas, de cuidar el bosque.
Por otro lado, los profesionales que elaboran planes no parecen encontrar la forma de otorgarles a éstos la entidad apropiada como herramientas para mejorar la rentabilidad empresarial y promover un manejo que conduzca a un bosque futuro de buena calidad. En este sentido, el profesional forestal debería ser, ante todo, un buen extensionista, que supiera comunicarse con todos los integrantes de la cadena de aprovechamiento e industrialización, para poder aprender de ellos y en la medida que fuese capaz de aportar una buena dosis de inteligencia e imaginación, plantear en la práctica una propuesta de manejo aceptable y viable tanto para el industrial como para el organismo fiscalizador. En esta circunstancia resulta indispensable instaurar un ámbito de profunda discusión entre profesionales, investigadores y fiscalizadores, a fin de consensuar propuestas de manejo silvícola factibles de aplicar en la práctica. En las actuales condiciones, los planes de manejo tienen una cuestionable eficacia como herramientas para mejorar la calidad del bosque actual y/o futuro $y$, al mismo tiempo, canalizar las expectativas del industrial traducidas en rentabilidad.

\section{AGRADECIMIENTOS}

A los ingenieros forestales Silvia Featherston, Fabio Berón y Gustavo Rôo, de la Dirección General de Bosques y Parques de Chubut, por habernos facilitado los planes de manejo para analizar y por sus valiosos comentarios en las numerosas oportunidades en que hemos tenido la posibilidad de mantener discusiones técnicas.

\section{BIBLIOGRAFIA}

(1) CARABELLI, F. Inventario Forestal del Bosque Nativo de la provincia del Chubut. Actas de las IV Jornadas Forestales Patagónicas. 1995. Tomo I. 160-169.

(2) DIRECCION GENERAL DE BOSQUES Y PARQUES DE LA PROVINCIA DEL CHUBUT. Inventario del bosque nativo de la provincia del Chubut. Subsecretaría de Desarrollo Económico, Ministerio de la Producción y Turismo, Gobierno de la Provincia del Chubut. 1997. 45 p.

(3) CHAUCHARD, L. Normas para la elaboración de planes de manejo y ordenación. Bases para su imple mentación. Proyecto de Desarrollo Forestal del Area Cordillerana. II Etapa. Consejo Federal de Inversiones. 1992. 28 p.

(4) BERON, F., H. ClAVERIE, V. POSTLER, S FEATHERSTON, G. RÔO. Regulación del rendimiento del bosque nativo productivo del Chubut. En: Actas Pri- 
BOSQUE 25(1): 3-13, 2004 Análisis de la eficacia de los planes de manejo de bosque nativo en la provincia de Chubut, Argentina

mer Congreso Latinoamericano IUFRO. Valdivia. Chile. 1998. Tema 3. 13 p.

(5) WILlIS, B. El Norte de la Patagonia. Naturaleza y Riquezas. Comisión de Estudios Hidrológicos. Dirección General de Ferrocarriles. Ministerio de Obras Públicas. Tomo I. EUDEBA. 1988. 500 p.

(6) ROTHKUGEL, M. Los Bosques Patagónicos. Oficina de Bosques y Yerbales. Dirección General de Agricultura y Defensa Agrícola. Ministerio de Agricultura. 1916. 207 p.

(7) HRANILOVIC, S., C. CORRALES. Delimitación de la Zona Forestal de la provincia del Chubut. Convenio Provincia del Chubut - Administración Nacional de Bosques. Informe inédito. 1966. $40 \mathrm{p}$.
(8) CARABELLI, F., H. CLAVERIE. Un análisis del estado de tenencia de la tierra y la importancia del inventario del bosque nativo en el marco de la planificación del uso del territorio en la provincia del Chubut. Seminario de Extensión Forestal. Departamento de Ingeniería Forestal. Facultad de Ingeniería, Univ. Nac. de la Patagonia. 1995. $17 \mathrm{p}$.

(9) TILING, M., J. JOVANOVSKI. Lenga (Nothofagus pumilio). Estudio de marketing de la Industria Patagónica. Publicación Técnica 16. Centro de Investigación y Extensión Forestal Andino-Patagónico. 1994. 54 p. 